\title{
Gamma ray bursts and gravitational waves: triggered search strategy in the LIGO science runs
}

\author{
S D Mohanty ${ }^{1,5}$, Sź Marka ${ }^{2}$, R Rahkola ${ }^{3}$, S Mukherjee ${ }^{1,5}$, I Leonor ${ }^{3}$, \\ R Frey ${ }^{3}$, J Cannizzo ${ }^{4}$ and J Camp ${ }^{4}$ \\ ${ }^{1}$ Max Planck Institut für Gravitationsphysik, Am Mühlenberg 1, Golm D14476, Germany \\ ${ }^{2}$ LIGO - California Institute of Technology, Pasadena, CA 91125, USA \\ ${ }^{3}$ University of Oregon, Eugene, OR 97403, USA \\ ${ }^{4}$ NASA/Goddard Space Flight Center, Greenbelt, MD 20771, USA
}

Received 31 August 2003

Published 9 February 2004

Online at stacks.iop.org/CQG/21/S765 (DOI: 10.1088/0264-9381/21/5/055)

\begin{abstract}
We describe a complete analysis pipeline for detecting and estimating gravitational wave signals in conjunction with observations of astrophysical phenomena with an electromagnetic or particle signature, examples of such phenomena being gamma ray bursts (GRB) and supernovae neutrino bursts. Our data analysis methods explicitly account for non-Gaussian and nonstationary features in real interferometric data. This pipeline is meant for signal searches in conjunction with individual GRB triggers and is being used for the analysis of data from the LIGO science runs (S1 and S2). However, many aspects of the pipeline are quite general and can also be used for other broadband interferometric detectors.
\end{abstract}

PACS numbers: $04.80 . \mathrm{Nn}, 95.55 . \mathrm{Ym}$, 95.85.Sz, 98.70.Rz

\section{Introduction}

Searches for gravitational wave (GW) signals can be performed in two ways: an untriggered search and an externally triggered search. The former method searches for GW signals with random arrival times. In the latter method, an astrophysical phenomenon, called a trigger, is first observed in a non-GW detector such as an optical telescope or neutrino detector. The search for GW signals is then restricted to an astrophysically motivated range of arrival times around a trigger time. In principle, a $\mathrm{GW}$ detector could also be used to trigger searches with other GW detectors.

Within the context of GW burst signal (GWB) searches, the sensitivity of the externally triggered method is potentially much higher than that of the untriggered method. (1) The restriction on GWB arrival times leads to a reduction in the false signal probability and, thus,

5 Present address: The University of Texas at Brownsville, 80 Fort Brown, Brownsville, TX 78520, USA. 
the detection threshold. (2) The non-GW observation can be used as a guide in selecting specific astrophysical models and, thus, optimizing the GWB search algorithm. The latter feature truly distinguishes a triggered search from other types of searches. Of course, the externally triggered method is necessarily limited to already observed phenomena while the untriggered method can turn up completely new ones.

Type II supernovae and gamma ray bursts (GRBs) are potentially strong sources of GWB emission in the ground-based detection band ( $\sim 10$ to $1000 \mathrm{~Hz})$. Both have an observable electromagnetic or particle signature and can, therefore, act as external triggers in a GW search. A new class of triggers called x-ray flashes (XRFs) have appeared starting with BEPPO-SAX observations [1]. Sources of XRFs are likely to be closely related to GRBs [2] and, hence, they are also potentially important GW emitters.

GRBs are detected at present at a rate of $\sim 1$ per day and all current astrophysical models suggest that the delay between a gravitational wave signal and the gamma ray signal is quite small $(\sim 0.1$ to $\sim 100 \mathrm{~s})$. In contrast, type II supernovae are detected at a much lower rate and the delay between the optical signature by which they are most easily detected and the gravitational wave signal is likely to be a few hours. From the point of view of developing an analysis pipeline, therefore, GRBs are more useful at the moment. A neutrino signal from a supernova should be nearly coincident in time with the GW signal. Our analysis, though developed for GRBs, would be directly applicable to such a trigger also. However, current neutrino detectors are limited in sensitivity and, hence, the rate of such high quality triggers is expected to be quite low over the foreseeable future. The usefulness of optical supernovae triggers will grow as interferometric detectors become more sensitive.

Among earlier works on GRB triggered GW searches, Piran and Kochanek [3] proposed using GRBs to trigger searches for GW signals from binary neutron star (NS) inspiral and mergers. However, an untriggered search for neutron star binary inspirals and mergers may have a better chance of detecting a signal since GRBs are almost certainly strongly beamed and those that point towards us will be more sparsely distributed. Besides, there is strong evidence now to exclude binary mergers as progenitors for long duration GRBs which form a substantial fraction of the total population. Finn et al [4] proposed an approach based on cross-correlation which requires little prior knowledge of progenitor models. A scheme was proposed whereby cross-correlated outputs from multiple GRB triggers can be combined to improve the collective signal to noise ratio. A multiple GRB trigger analysis for binary inspiral searches was investigated in [5]. Multi-trigger analyses in the context of resonant mass detectors have been investigated by [6-8].

The aim of the analysis pipeline described here is to search for GW signals in coincidence with individual GRBs. An important feature of this pipeline is that it is geared towards the analysis of real interferometer data with all their attendant complications such as lines and non-stationarity. We use a sophisticated approach to cross-correlation in which we scan a range of free parameters. We use current astrophysical theories to limit the ranges of these parameters [9].

There are several parts to our analysis pipeline. First comes the collection of triggers (we focus on GRBs at the moment). Next the interferometric dataset to use for searches around the triggers is fixed. This dataset consists of a pair of interferometer outputs in two parts, on-source and off-source data. The former are a stretch of data near the trigger in which a potential GW signal exists while the latter are data that are unlikely to contain such a signal. The dataset is preprocessed (or conditioned) and then fed into our signal search algorithm. The final results must then be converted into astrophysically meaningful ones. 
Our search algorithm uses off-source data to optimize parameters of conditioning, to measure sensitivity to various burst waveforms, to measure the expected false alarm rate and to standardize statistics derived from the on-source data.

From the on-source data we generate cross-correlations over a region of our search parameter space. We use three different test statistics at this stage to process the multidimensional output and generate potential candidate events. After setting a detection threshold based on an estimated false alarm rate, the sensitivity for a large number of simulated and astrophysically motivated waveforms is measured via software and hardware injections. If no candidate events are found above the detection threshold, an upper limit can be set.

The development of our search algorithm has been guided by our experience with real and realistic data. Often, gravitational wave data analysis algorithms are designed or characterized for the ideal case of Gaussian and stationary noise. However, if special care is not taken to account for features of real data such as non-stationarity an algorithm developed for ideal noise may perform quite poorly with real data. Our algorithms have, on the other hand, been designed to handle features of real data and are constructed to be robust. As a consequence, we have many design choices in algorithm construction as opposed to the Gaussian, stationary noise case where an optimum algorithm is usually straightforward to construct theoretically.

Currently, significant amount of realistic data is available from the LIGO detectors, either as playground segments from science runs or good quality commissioning data from the 'freeze' periods before the science runs. Also, an effort to generate simulated noise with realistic properties (including non-stationarity) modelled after real data is under way [10]. Hence, there are plenty of real and realistic data that can be used to validate our design choices.

The robust approach to algorithm design has led us to a method with good sensitivity in real data for short burst waveforms where the signal shape is unknown, but the source direction is known. The method can also be modified for sources with unknown direction, though the relative degradation in performance due to an increased false alarm rate remains to be measured.

In this paper, we describe the present form of our analysis pipeline and present preliminary measures of sensitivity using simple simulated noise and signals. The detailed description and performance characterization with real interferometer data will have to be the material of a future paper.

\section{Collection of triggers}

LIGO has connections to two major networks specializing in distribution of GRB observational data from various sources from which we receive timely notices through the internet. LIGO is also connected to the supernova early warning system (SNEWS) [11].

The GRB coordinates network (GCN) [12] is an internet-based, near real-time distributor of GRB and related notices, compiling observations from several observatories and satellites. It also serves as a distributor of follow-up reports supplied by the GRB community. The third interplanetary network (IPN3) relies on a system of orbiting spacecraft equipped with GRB detectors. IPN3 derives the source direction information via triangulation, utilizing the varying arrival time of the burst at each spacecraft. Reports are available via the internet several days after the burst. Recently, the IPN3 has been circulating its notices through the GCN.

The GCN notices are automatically parsed and relevant information such as sky direction and time of event are extracted and stored. This information can also be stored in an LDAS 
(Ligo data analysis system) database. A system has been implemented to keep the LIGO controllers informed of triggers so that lock status can be maintained or extended.

The trigger information is then translated into quantities that are relevant to our data analysis algorithms. These are as follows.

Propagation time delay between interferometers. The propagation time delay is the projection of the vector connecting the two interferometers, $L_{H 2-L 1}$, on the unit vector pointing to the trigger sky position.

Internal time shift between interferometers. In addition to the arrival time difference due to the XRF source location relative to the Earth, we have to account for corrections from the LIGO timing system.

Effects of spacecraft location on start time. We calculate the delay between the arrival time of the GRB at the spacecraft and the L1 interferometer. This is a negligible correction for current GRB triggers.

Signal attenuation factor. The LIGO interferometers do not have completely isotropic antenna patterns. Thus, the amplitude of the observed signal depends on its orientation with respect to each of the interferometers. We use this information along with estimates of sky position error to assign the quality of a trigger.

\section{Selection of datasets}

As mentioned in section 1, our dataset consists of pairs of interferometer outputs which is further demarcated into on-source and off-source data. We use off-source data to optimize the free parameters of the search algorithm, to measure sensitivity to various burst waveforms, to measure the expected false alarm rate and to standardize the statistics derived from the on-source data. Though, in principle, the off-source data can be collected from any stretch including ones that belong to different lock periods, we restrict ourselves to the same lock stretch as the one containing the on-source data.

The on-source data consist of a segment from each interferometer. Let $t_{\gamma}^{(\mathrm{A})}$ be the time of arrival of the GRB at interferometer A, say, and $t_{\gamma}^{(\mathrm{B})}$ be the same for interferometer B. The propagation time delay is $\tau_{\mathrm{AB}}=t_{\gamma}^{(\mathrm{B})}-t_{\gamma}^{(\mathrm{A})}$. Let the segment from A be chosen such that it lasts from time $t=t_{1}$ where $t_{1} \leqslant t_{\gamma}^{(\mathrm{A})}$, to $t=t_{2}$ where $t_{2} \geqslant t_{\gamma}^{(\mathrm{B})}$. Then the segment from $\mathrm{B}$ is chosen such that it lasts from $t=t_{1}+\tau_{(\mathrm{AB})}$ to $t=t_{2}+\tau_{\mathrm{AB}}$. Since the delay between the GRB trigger and the GW signal is intrinsic to the source, this also ensures that the GW signals in each segment line up. However, the offset of the GW signal from the segment origin is unknown a priori. Most astrophysical models suggest that the GW signal is emitted either before or simultaneously with the GRB trigger so that it would be appropriate to choose $t_{1}<t_{\gamma}^{(\mathrm{A})}$ and $t_{2}=t_{\gamma}^{(\mathrm{A})}$. However, we also allow for astrophysical models that are exceptions [13] for which $t_{2}^{(\mathrm{A})}>t_{\gamma}^{(\mathrm{A})}$.

\section{Data conditioning}

Raw interferometric data must be conditioned in order to achieve the best sensitivity for our search algorithms. For our algorithms, the most beneficial conditioning is to remove high power narrowband noise sources or lines from the data. The detrimental effect of lines on cross-correlation based searches has been studied in [14]. Next, the data are bandpass filtered to (1) throw away the extremely strong seismic noise at low frequencies and to (2) throw away 
higher frequencies where no astrophysical models predict a GW signal from GRBs while the noise power continues to rise.

Finally, we would like the cleaned and bandpassed data to have a flat power spectral density in the band of interest. These are called whitened data. Among other things, whitening simplifies the theoretical analyses of algorithms. If the noise were stationary, whitening entails no loss of performance and it can be implemented via a suitably designed linear time invariant whitening filter. Strictly speaking, power spectral density is an undefined quantity for non-stationary noise but the notion can be used in an approximate sense there also. Correspondingly, non-stationary noise can be approximately whitened which is also beneficial.

We use a pre-processing procedure developed in collaboration with [15] that combines all the steps mentioned above. However, alternative methods are available for data whitening $[16,18]$ as well as the removal of lines [19-22]. We intend to test some of these alternative methods in the context of a triggered search in the future.

Ideally, each interferometer output should be calibrated to have units of strain as the first step of conditioning, but there are several practical problems involved. However, we use whitened data and since a cross-correlation can be constructed out of the products of discrete Fourier transforms [23], we only need the difference in calibration phases. Therefore, a time domain filter is constructed that applies the proper phase difference calibration to one of the interferometer outputs.

\section{Search algorithm}

\subsection{Overview}

As indicated earlier, our search algorithm is based on the cross-correlation of pairs of interferometer outputs. Cross-correlation based methods are well suited for the problem of signals having a priori unknown waveforms that are observed by multiple detectors. However, we do not use the cross-correlation itself as a test statistic. Instead we transform our pair of raw interferometer outputs into a multi-dimensional field built out of cross-correlations generated over a space of free parameters (see below). It is this multi-dimensional transform that is then processed as explained below.

We use non-parametric tests, carefully normalized cross-correlations and local measures of deviation from the expected behaviour. There is, of course, considerable freedom in the choices we need to make with respect to the above. We, therefore, validate the effectiveness of our design choices using real or realistic interferometric data. Simulations with Gaussian stationary noise are of very limited use for this purpose and are avoided. We do, however, run the final pipeline on Gaussian stationary noise to see how much our sensitivity is worsened by the necessity of taking real data into account. This does not mean, and this should be emphasized, that a method that was optimal for Gaussian stationary noise would necessarily perform better than our algorithm in real noise.

\subsection{Basic definitions and notation}

Let the two interferometric time series be $x_{i}[k], i=1,2$ where $k$ corresponds to the time index, $k=0,1, \ldots, N-1$. Recall from section 3 , that a relative temporal shift corresponding to the propagation time delay (cf section 2) is already present at this stage.

Let $y_{i}[k ; \delta, M], k=0, \ldots, M-1$, be a segment of $x_{i}[k]$ such that,

$$
y_{i}[k ; \delta, M]=x_{i}[k+\delta] \text {. }
$$


Let $\mu_{i}(\delta, M)$ be the sample mean of $y_{i}[k ; \delta, M]$,

$$
\mu_{i}(\delta, M)=\frac{1}{M} \sum_{n=0}^{M-1} y_{i}[n ; \delta, M]
$$

Let,

$$
\Sigma_{i}(\delta, M)=\sum_{n=0}^{M-1}\left(y_{i}[n ; \delta, M]-\mu_{i}(\delta, M)\right)^{2} .
$$

Let $\chi^{\prime}\left(\delta_{1}, \delta_{2}, M\right)$ be the ordinary cross-correlation with lag $\delta_{1}-\delta_{2}$ between pairs of segments $y_{1}\left[k ; \delta_{1}, M\right]$ and $y_{2}\left[k ; \delta_{2}, M\right]$,

$\chi^{\prime}\left(\delta_{1}, \delta_{2}, M\right)=\frac{1}{M} \sum_{n=0}^{M-1}\left(y_{1}\left[n ; \delta_{1}, M\right]-\mu_{1}\left(\delta_{1}, M\right)\right)\left(y_{2}\left[n ; \delta_{2}, M\right]-\mu_{2}\left(\delta_{2}, M\right)\right)$.

We call $M$ the integration length for the cross-correlation.

We derive a more complex indicator of cross-correlation from $\chi^{\prime}$,

$\chi\left(\delta_{1}, \delta_{2}, M, K\right)=\frac{\chi^{\prime}\left(\delta_{1}, \delta_{2}, M\right)}{\sqrt{\frac{\Sigma_{1}\left(\delta_{1}-(M+K), K\right)+\Sigma_{1}\left(\delta_{1}+M, K\right)}{2 K}} \sqrt{\frac{\Sigma_{2}\left(\delta_{2}-(M+K), K\right)+\Sigma_{2}\left(\delta_{2}+M, K\right)}{2 K}}}$.

Essentially $\chi\left(\delta_{1}, \delta_{2}, M, K\right)$ is the same as the well-known cross-correlation coefficient [24],

$$
\frac{\chi^{\prime}\left(\delta_{1}, \delta_{2}, M\right)}{\sqrt{\frac{\Sigma_{1}\left(\delta_{1}, M\right)}{M}} \sqrt{\frac{\Sigma_{2}\left(\delta_{2}, M\right)}{M}}}
$$

but instead of the normalization in the denominator constructed out of the same segment as the numerator, we use segments local to but different from the numerator segments. The length, $K$, of the segments used in normalization is, in general, larger than $M$ and is a parameter that is fixed by hand once at the beginning of a search. We choose $K=2 M$. In the following we drop this parameter from our expressions,

$$
\chi\left(\delta_{1}, \delta_{2}, M, K\right) \equiv \chi\left(\delta_{1}, \delta_{2}, M\right) .
$$

The indicator $\chi\left(\delta_{1}, \delta_{2}, M\right)$ has the property of being (nearly) scale invariant like the crosscorrelation coefficient. Hence, it is more robust against non-stationary variance of the noise. However, the latter has the undesirable property of putting the signal in the normalization factor which reduces the signal to noise ratio for strong signals. The indicator $\chi\left(\delta_{1}, \delta_{2}, M\right)$ is designed to avoid this problem.

\subsection{Parameter scan}

The indicator $\chi\left(\delta_{1}, \delta_{2}, M\right)$ depends on three free parameters: the offset from origin $\delta=\delta_{1}$, the lag $\tau=\delta_{1}-\delta_{2}$ and the integration length $M$. The optimum values for these parameters depend not only on the duration of the signal but also on the amplitude of the signal [25]. It is therefore necessary, especially because of the latter, that several different values for these parameters be used in our search. The range of the parameters $\delta, M$ and $\tau$ can be fixed based on plausible astrophysical models of GRBs.

The next logical step in the search algorithm then is to scan the parameter space, i.e., to generate a multi-dimensional transform, $S(\delta, \tau, M)$, defined as

$$
S(\delta, \tau, M)=\chi(\delta, \delta+\tau, M) .
$$

We call $S(\delta, \tau, M)$ the $S$-volume. 


\subsection{Collapsing the S-volume}

Information about a potential signal is hidden in the S-volume and needs to be collapsed into a test statistic that can be compared with a detection threshold.

First, we need to collapse the $S$-volume to a two-dimensional plane. The plane $S(\delta, 0, M)$, called a corrgram [26], is special in the sense that the signal-signal cross-correlation is maximized at the appropriate values of $\delta$ and $M$. However, the corrgram is just one section of the $\mathrm{S}$-volume and it does not make the best use of potentially useful information along the $\tau$ direction. We have explored three semi-independent ways to collapse the S-volume (by one dimension) that have better performance than the corrgram. These lead to correspondingly different test statistics as follows.

First, there is a common step involved. For fixed $\delta$ and $M$, we smooth (the absolute value of) the sequence in $\tau,|C(\tau ; \delta, M)|=|S(\delta, \tau, M)|$, by convolving it with a Gaussian kernel of fixed width which is smaller than the signal width that integration length (i.e., $M$ ) is sensitive to. We continue to denote the smoothed sequence by $C(\tau ; \delta, M)$. Smoothing allows easy extraction of the following properties:

- the maximum value $C_{\max }(\delta, M)$ of $C(\tau ; \delta, M)$;

- the location $T_{\max }(\delta, M)$ of the maximum $\left(C\left(T_{\max } ; \delta, M\right)=C_{\max }(\delta, M)\right.$;

- a range of $\tau$ centred at $T_{\max }$ called the main lobe;

- the range of $\tau$ remaining after taking out twice the main lobe is called the side lobe.

We then collapse the S-volume into a two-dimensional plane in the following different ways.

LocPeak or 'S' method.

$$
S(\delta, \tau, M) \rightarrow S_{S}(\delta, M)=C_{\max }(\delta, M) .
$$

It is expected that in the case of coincident signals and random noise, $C(0 ; \delta, M)$ would be much more pronounced, compared to say $C(\tau \gg M ; \delta, M)$. For uncorrelated noise there should not be any enhancement around the expected time lag of $\tau=0$. This allows discrimination between signals and noise. As this relative quantity is strictly computed based on local data, it is fairly insensitive to effects due to slow non-stationarity.

FineLag or ' $\mathrm{T}$ ' method.

$$
S(\delta, \tau, M) \rightarrow S_{T}(\delta, M)=T_{\max }(\delta, M) .
$$

For external triggers with a well-known direction we correct for the propagation time delay by introducing a time shift between interferometers. In practice, it means that the maximum of the smoothed cross-correlation must occur very close to the expected time lag, $\tau=0$, for real coincident signals. For non-correlated noise this requirement does not exist, therefore we should expect no enhancement for any specific favoured time lag there. Therefore, $T_{\max }$ is an independent measure of the presence of a real coincident signal.

LocStat or ' $\mathrm{K}$ ' method.

$$
S(\delta, \tau, M) \rightarrow S_{K}(\delta, M) .
$$

When there is a coincident signal, the statistical behaviour of the cross-correlation function will be different in the main lobe when compared to the distributions in the side lobe. For uncorrelated noise there should not be any difference. We use the non-parametric two sample Kolmogorov-Smirnov test between the sets of (unsmoothed) samples of $C(\tau ; \delta, M)$ from the main and side lobes. This method is somewhat comparable to the one used in $[7,27]$. However, there is a very significant difference in that, instead of relying on the comparison between data 
and expected Gaussian behaviour, our test compares locally obtained distributions. It is likely that this is useful when considering real data but disadvantageous when considering close to Gaussian data.

The three different collapses $S_{S}, S_{T}$ and $S_{K}$ are not readily comparable. One can of course standardize these two-dimensional maps fairly easily. This is the next step.

\subsection{Standardization of collapsed volume}

We estimate the distribution of $S_{S}(\delta, M)$ from off-source data for each value of $M$. These distributions are fairly stationary over different segments of the interferometer output $x_{i}[k]$ since the individual collapses were constructed to be resistant to non-stationarity. Based on these measured distributions one can easily convert the values of the on-source (and off-source) $S_{S}(\delta, M)$, for a fixed $M$, into a significance value. That is, the probability, as estimated from the measured distribution, of the value of $S_{S}(\delta, M)$ is greater than or equal to the observed value. The same procedure is repeated for $S_{T}$ and $S_{K}$ planes. After transformation to significance values, the $S_{S}, S_{T}$ and $S_{K}$ planes are compatible and can be combined.

\subsection{Event identification}

After the standardization stage, a threshold is put and only the values of the collapsed planes above the threshold are retained while the rest are set to zero. Finally the two-dimensional plane $S_{S}$ (or $S_{T}, S_{K}$ ) is collapsed into a one-dimensional sequence $R_{S}(\delta)$ (or $R_{T}, R_{K}$ ) by integrating the number of surviving values along $M$ for a range of values of $\delta$. The maximum of this one-dimensional sequence is then chosen as our test statistic $\Lambda_{S}$ (or $\Lambda_{T}, \Lambda_{K}$ ).

We can measure the covariance between the comparably sensitive $\Lambda_{S}$ and $\Lambda_{K}$ test statistics. The measured covariance matrix can then be diagonalized, thus yielding the linear combinations of $\Lambda_{S}$ and $\Lambda_{K}$ which are uncorrelated. These linear combinations are then combined in quadrature. Finally, $\Lambda_{T}$ can be used as a veto on the combined test statistic.

\section{Performance in Gaussian noise}

A Monte Carlo simulation was carried out to study false alarm rate versus detection efficiency in ideal Gaussian and stationary noise. For each trial, pairs of Gaussian stationary noise sequences were generated with a sampling frequency of $16384 \mathrm{kHz}$ (corresponding to fast channels in LIGO). The rms of the noise sequences were set to be the same as estimated from stretches of real data from the S2 run of the LIGO Hanford $2 \mathrm{~km}$ and $4 \mathrm{~km}$ detectors. (The real data from each detector were bandpass filtered (250 to $1000 \mathrm{~Hz}$ ) before estimating the rms.) Gaussian modulated sinusoidal signals with varying peak amplitudes were injected into the simulated noise.

The dataset was then passed through exactly the same pipeline as we would use for a real search. Figure 1 shows the detection efficiency as a function of the peak amplitude of the injected signal. Further details are mentioned in the caption of the figure.

\section{Discussion}

We have described a complete end to end analysis pipeline for carrying out a triggered search for gravitational wave burst signals. The search algorithm at the core of this pipeline is designed to handle complications in real data such as the presence of high power line features 


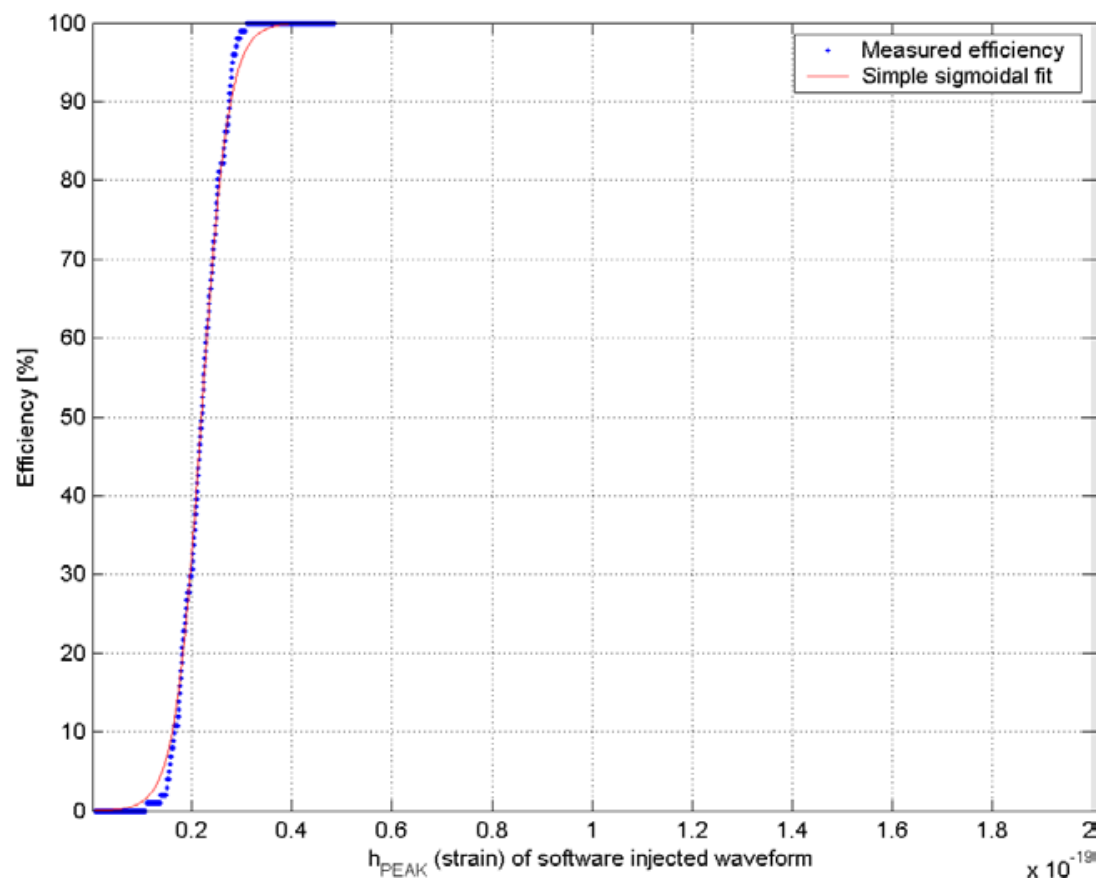

Figure 1. Detection efficiency versus peak amplitude. Sine-Gaussian signals with carrier frequency of $361 \mathrm{~Hz}$ and envelope FWHM of $1 \mathrm{~ms}$. The estimated false alarm rate was less than $30 \mathrm{mHz}$.

and non-stationarity. The algorithm uses a sophisticated approach based on cross-correlations computed over a three-dimensional parameter space. The information contained in this multidimensional volume is collapsed to a two-dimensional plane using three semi-independent methods (LocPeak, LocStat, FineLag), followed by a second collapse to a single test statistic. We find the performance of this algorithm to be very good in real data.

A follow-up to our current search strategy will be to combine the results obtained from the comparably sensitive LocPeak and LocStat methods. As these variables are not necessarily independent when considering random, uncorrelated noise one has to measure out the covariance matrix and diagonalize it to derive independent quantities as linear combinations of the LocPeak and the LocStat variables. The resulting quantities can be combined in quadrature. The significant clusters produced should then be vetoed based on the results of the FineLag method (i.e., veto them if the measured time lag is far from the expected time lag as compared to lags occurring in background data). The surviving candidates can then be further scrutinized by evaluating their signal shape, relative magnitude ratio and frequency content. At this point possible environmental and instrumental vetoes must be also considered to veto possible candidates.

With several currently operating GRB detectors (HETE2 [28], INTEGRAL [29]) and several planned ones (SWIFT [30], GLAST [31]), one can be assured of collecting 1000 or more GRB triggers over the next few years. A substantial number of these triggers will also have measured redshifts along with information about possible progenitors gleaned from afterglow observations, which would allow us to perform some selection on the trigger quality. It is therefore imperative that use be made of these triggers in conjunction with data from the large scale GW interferometers which will attain stable performance levels over the same timescale. 


\section{Acknowledgments}

We thank members of the LIGO Science Collaboration, in particular members of the Bursts Upper Limit group, for very helpful comments and suggestions. We thank the LIGO laboratory for making data from the Science runs available to us.

\section{References}

[1] Heise J et al 2001 2nd Rome Workshop: Gamma Ray Bursts in the Afterglow Era ed N Masseti et al (Preprint astro-ph/0102277)

[2] Kippen R M et al 2002 Preprint astro-ph/0203114

[3] Kochanek C and Piran T 1993 Astrophys. J. Lett. 41717

[4] Finn L S, Mohanty S D and Romano J 1999 Phys. Rev. D 60121101

[5] Bonazzola P and Gourgoulhon S 1998 Preprint astroph/9801162

[6] Murphy M T et al 2000 Mon. Not. R. Astron. Soc. 316657

[7] Modestino G and Moleti A 2002 Phys. Rev. D 66022005

[8] Astone P et al 2002 Phys. Rev. D 65102002

[9] Frey R 2003 LIGO Technical Note LIGO-T030274-00-Z

[10] Mukherjee S 2003 LIGO Technical Note LIGO-G030374-00-Z

[11] Scholberg K 2000 Proc. 3rd Eduardo Amaldi Conf. (AIP Conf. Proc. vol 523) ed S Meshkov (New York: AIP) p 355

[12] Barthelmy S D et al 2000 Gamma Ray Bursts, 5th Huntsville Symp. (AIP Conf. Ser. vol 526) ed R M Kippen, R S Mallozi and G J Fishman (Melville, NY: AIP) p 731

[13] van Putten M H P M et al 2003 Preprint gr-qc/0308016 (Phys. Rev. D at press)

[14] Rahkola R et al 2003 LIGO Technical Note LIGO-T030050-00-Z

[15] Chatterji S 2003 LIGO Technical Note LIGO-G030439-00-Z

[16] Cuoco E et al 2001 Class. Quantum Grav. 18 1727-52

[17] Cuoco E et al 2001 Phys. Rev. D 64122002

[18] Mukherjee S 2003 Class. Quantum Grav. 20 S925

[19] Sintes A M and Schutz B 1998 Phys. Rev. D 58122003

[20] Finn L S and Mukherjee S 2001 Phys. Rev. D 63062004

[21] Finn L S et al 2000 Proc. 3rd Eduardo Amaldi Conf. (AIP Conf. Proc. vol 523) ed S Meshkov (New York: AIP) p 451

[22] Mohanty S D 2002 Class. Quantum Grav. 19 1513-9

[23] Bracewell R N 1999 The Fourier Transform and its Applications 3rd edn (New York: McGraw-Hill)

[24] Anderson T W 1984 An Introduction to Multivariate Statistical Analysis 2nd edn (New York: Wiley)

[25] Mohanty S D et al 2003 LIGO Technical Note LIGO-T030113-00-D

[26] Mohanty S D et al 2003 LIGO Technical Note LIGO-T030114-00-D

[27] Cadonati L 2003 LIGO Technical Note LIGO-G030438-00-Z

[28] Ricker G R et al 2003 American Astronomical Society Meeting 20254.01

[29] Parmar A N et al 2003 X-ray and gamma ray telescopes and instruments for astronomy Proc. SPIE 4851

[30] Geherels N et al 2003 American Astronomical Society Meeting 20248.02

[31] Burnett T H et al 2003 American Astronomical Society Meeting 201131.01 